\title{
Astrocytes Enhance Streptococcus suis-Glial Cell Interaction in Primary Astrocyte-Microglial Cell Co-Cultures
}

\author{
Jana Seele $^{1,2,3}$, Roland Nau ${ }^{2,3}$, Chittappen K. Prajeeth ${ }^{4}$, Martin Stangel ${ }^{4}$, \\ Peter Valentin-Weigand ${ }^{1}$ and Maren Seitz ${ }^{1, *}$ \\ 1 Center for Infection Medicine, Institute for Microbiology, University of Veterinary Medicine Hannover, \\ Bischofsholer Damm 15, Hannover 30173, Germany; jana_seele@gmx.de (J.S.); \\ peter.valentin@tiho-hannover.de (P.V.-W.) \\ 2 Institute for Neuropathology, University Medical Center Göttingen, Robert-Koch-Straße 40, Göttingen 37099, \\ Germany; rnau@gwdg.de \\ 3 Department of Geriatrics, Evangelisches Krankenhaus Göttingen-Weende, An der Lutter 24, \\ Göttingen 37075, Germany \\ 4 Department of Neurology, Center for Systems Neuroscience (ZSN), Hannover Medical School, \\ Carl-Neuberg-Straße 1, Hannover 30625, Germany; ChittappenKandiyil.Prajeeth@mh-hannover.de (C.K.P.); \\ Stangel.Martin@mh-hannover.de (M.S.) \\ * Correspondence: maren.seitz@tiho-hannover.de; Tel.: +49-511-856-7217; Fax: +49-511-856-7697
}

Academic Editor: Lawrence S. Young

Received: 1 May 2016; Accepted: 7 June 2016; Published: 13 June 2016

\begin{abstract}
Streptococcus (S.) suis infections are the most common cause of meningitis in pigs. Moreover, S. suis is a zoonotic pathogen, which can lead to meningitis in humans, mainly in adults. We assume that glial cells may play a crucial role in host-pathogen interactions during S. suis infection of the central nervous system. Glial cells are considered to possess important functions during inflammation and injury of the brain in bacterial meningitis. In the present study, we established primary astrocyte-microglial cell co-cultures to investigate interactions of $S$. suis with glial cells. For this purpose, microglial cells and astrocytes were isolated from new-born mouse brains and characterized by flow cytometry, followed by the establishment of astrocyte and microglial cell mono-cultures as well as astrocyte-microglial cell co-cultures. In addition, we prepared microglial cell mono-cultures co-incubated with uninfected astrocyte mono-culture supernatants and astrocyte mono-cultures co-incubated with uninfected microglial cell mono-culture supernatants. After infection of the different cell cultures with $S$. suis, bacteria-cell association was mainly observed with microglial cells and most prominently with a non-encapsulated mutant of $S$. suis. A time-dependent induction of NO release was found only in the co-cultures and after co-incubation of microglial cells with uninfected supernatants of astrocyte mono-cultures mainly after infection with the capsular mutant. Only moderate cytotoxic effects were found in co-cultured glial cells after infection with S. suis. Taken together, astrocytes and astrocyte supernatants increased interaction of microglial cells with S. suis. Astrocyte-microglial cell co-cultures are suitable to study S. suis infections and bacteria-cell association as well as NO release by microglial cells was enhanced in the presence of astrocytes.
\end{abstract}

Keywords: astrocytes; microglial cells; co-cultures; bacteria-cell-association; NO release; S. suis

\section{Introduction}

Streptococcus (S.) suis is one of the most important porcine pathogens causing meningitis, arthritis, endocarditis, in some cases encephalitis and other pathologies [1,2]. Moreover, it is a zoonotic pathogen. Most human infections occur in Southeast Asia with meningitis as the main pathology [3]. 
S. suis possesses a variety of virulence and virulence-associated factors including the capsule (CPS) and suilysin [4]. The capsule was shown to protect $S$. suis against killing by phagocytes and deposition of complement [5-8]. Moreover, in pig infection experiments capsular mutants of S. suis were completely avirulent [6]. Suilysin, the hemolysin of S. suis, is a pore-forming cholesterol-dependent cytolysin and might support $S$. suis to cross epi- and endothelial barriers $[9,10]$.

To cause meningitis $S$. suis has to enter the central nervous system (CNS) via the blood brain barrier (BBB) or the blood cerebrospinal fluid barrier (BCSFB) [9]. Adhesion to and invasion of brain microvascular endothelial cells (part of the $\mathrm{BBB}$ ) and cells of the plexus chorioideus (part of BCSFB) by S. suis were shown in vitro [11-15]. Astrocytes form together with endothelial cells the $\mathrm{BBB}$ and separate the neuronal parenchyma from non-neuronal cells along the blood vessels and the meninges [16]. Besides providing structural support and nutrients for neuronal cells, [17] astrocytes have barrier functions, liming the spread of infections to the CNS parenchyma, and have pro- as well as anti-inflammatory properties [16]. Although it is hypothesized that astrocytes play a crucial role in host-pathogen interaction during streptococcal meningitis, interactions of streptococci and astrocytes are only poorly investigated [18]. A further glial cell subtype, the microglial cells, represents macrophages of the CNS, which play an important role as phagocytic and antigen-presenting cells [19]. It has been described that activation of microglial cells is modulated by astrocytes [20] and astrocytes are necessary for activation of microglial cells in co-culture e.g., during borna virus infection [21]. Moreover, both cell types respond to bacterial infections of the CNS [22-24], have direct contact in brain tissue, and were shown to interact through signaling in cell culture $[25,26]$.

Interaction of S. suis with human astrocyte and microglial cell lines as well as with primary murine astrocytes has been previously reported, and an involvement of these cell types in S. suis infections of the CNS was shown [27-30], but so far primary astrocyte and microglial cell co-cultures were not studied. Co-cultures enable analysis of $S$. suis interactions with and between those most abundant and important cell types of the CNS. A further advantage of a murine primary co-culture system is the use of cells from genetically modified animals. For that reason the aim of this study was to establish murine primary astrocyte microglial cell co-cultures for $S$. suis infections and to compare interaction of S. suis with mono- and co-cultured astrocytes and microglial cells.

\section{Results and Discussion}

\subsection{Association of S. suis with Primary Astrocytes and Microglial Cells}

For analysis of S. suis-glial cell interactions, primary astrocytes and microglial cells from brain tissue of new-born C57BL/ 6 mice were characterized by flow cytometry analysis. As shown in Figure 1A astrocytes were positive for the astrocyte cell-surface marker ACSA-2 as well as for the main intermediate filament protein of mature astrocytes (glial fibrillary acidic protein, GFAP). Other classical cell-surface marker proteins (CD11b, CD11c, and CD45) and the cytosolic protein CD68 of immune cells, including microglial cells, were rarely detectable. In contrast, microglial cells (Figure 1B) expressed all common immune cell markers and the transmembrane chemokine receptor CX3CR1, which was absent in astrocytes. Both, GFAP and ACSA-2-positive cells were found in low amounts in microglial cell preparations. Glial cell preparations showing less than $20 \%$ contamination of the respective other glial cell type were defined as pure.

To further study S. suis-glial cell interactions, pure astrocyte and microglial cell preparations were used in various cell culture systems. At first, astrocytes and microglial cells were maintained separately in mono-cultures. In addition, astrocyte mono-cultures were pre-incubated with supernatants of uninfected microglial cell cultures and microglial mono-cultures were pre-stimulated with supernatants of uninfected astrocyte mono-cultures, respectively. At last, astrocytes and microglial cells were combined in co-cultures using two different amounts of microglial cells. After one day of cultivation co-cultures reached a final microglial cell:astrocyte ratio of 1:2 (low amount of microglial cells) and 1:1 (high amount of microglial cells), respectively. These various glial cell cultures were infected 
with carboxyfluorescein succinimidyl ester (CFSE)-stained S. suis serotype 2 wildtype (wt) strain 10, its non-encapsulated mutant strain $10 \operatorname{cps} \triangle E F$ and a suilysin-deficient strain $10 \Delta$ sly, respectively, at a multiplicity of infection (MOI) of 10:1 for $2 \mathrm{~h}$. Afterwards glial cells were harvested and CFSE-positive cells were quantified by flow cytometry. In all mono-cultured glial cell cultures, irrespectively of the pretreatment with uninfected glial cell supernatants, only the non-encapsulated strain showed a significant bacteria-cell association (Figure 2A-D). Higher association rates were found in microglial cell mono-cultures (Figure 2B; 19.6\%) than in astrocyte mono-cultures (Figure 2A; 6.9\%). Nevertheless, pretreatment of microglial cell mono-cultures with supernatants of uninfected astrocyte mono-cultures increased the association rate of $10 \mathrm{cps} \triangle E F$ to $28.7 \%$ (Figure 2D). A comparable number of CFSE-positive cells (Figure 2E; $28.6 \%$ ) was found in the 10cps $\triangle E F$-infected astrocytes-microglial co-culture with low amount of microglial and the highest bacteria-cell association for $10 c p s \Delta E F$ was observed in the co-culture with a high amount of microglial cells (Figure 2F; 41.6\%). In contrast, both encapsulated S. suis strains (strain 10 and $10 \Delta$ sly) showed only in the co-cultures a lower but significant and comparable glialcell association (Figure 2E,F). To further elucidate the preferred cell type for interaction, S. suis-infected glial cells were additionally stained after harvesting with the cell-surface markers ACSA-2 and CX3CR1 and analyzed by flow cytometry.
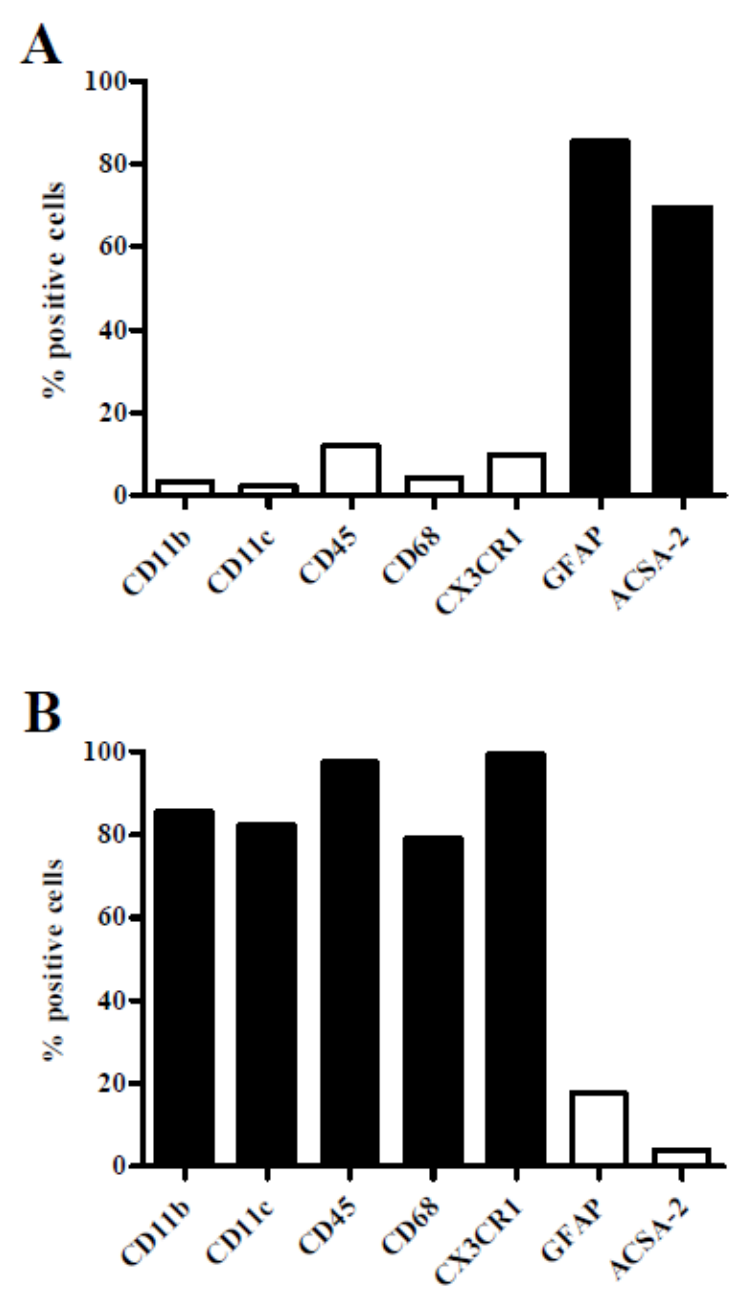

Figure 1. Phenotypical characterisation of primary mouse astrocytes and microglial cells. Flow cytometry analysis of primary astrocytes (A) and microglial cells (B) isolated from brain tissue of new-born C57BL/ 6 mice. Cells were stained for cell-surface antigens (CD11b, CD11c, CD45, CX3CR1, and ACSA-2) and intracellular antigens (CD68 and GFAP) as indicated. Means of two independent preparations are shown. 


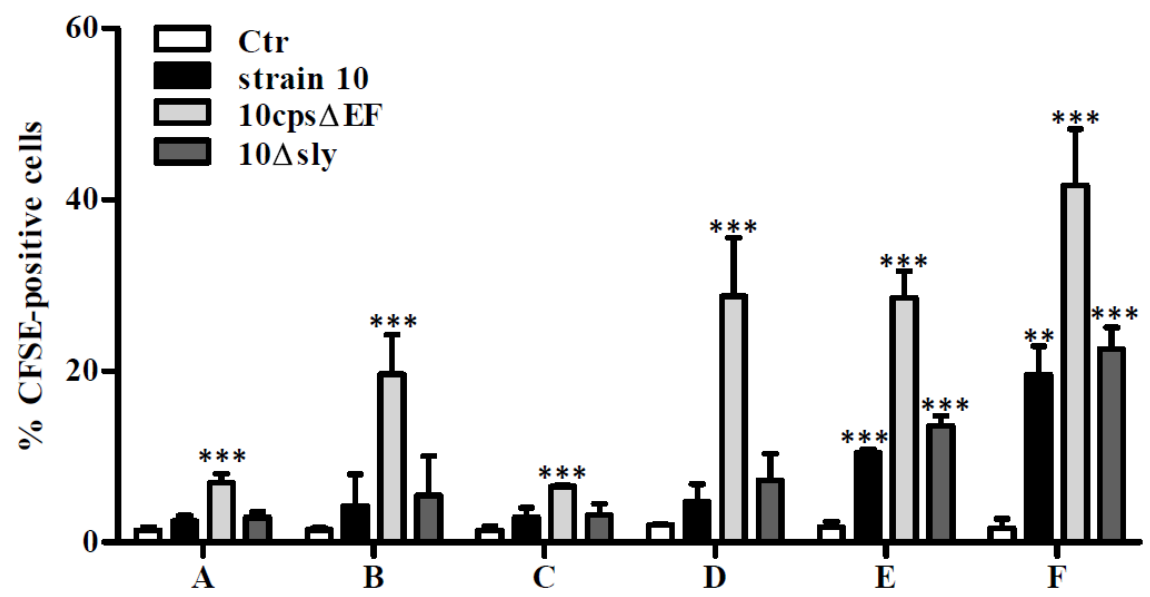

Figure 2. Association of $S$. suis with primary mouse glial cells. Various glial cell culture systems: (A) astrocyte mono-culture, (B) microglial cell mono-culture, (C) astrocyte mono-culture pre-incubated with supernatants (SN) of uninfected microglial cell cultures, (D) microglial cell mono-culture pre-incubated with SN of uninfected astrocyte cultures, (E) astrocyte-microglial cell co-culture (low amount of microglial cells), and (F) astrocyte-microglial cell co-culture (high amount of microglial cells), respectively, were infected with CFSE-labeled S. suis strain 10,10cps $\Delta E F$, or 10 $\Delta$ sly at a MOI of 10:1. Percentage of CFSE-positive cells were measured by flow cytometry. Results are expressed as means with standard deviation (SD) of three independent experiments, and statistically significant differences when compared to uninfected control cells are indicated by ** $(p$-value $<0.01)$, and ${ }^{* * *}(p$-value $<0.001)$, one-way analysis of variance (ANOVA) followed by a Dunnett post-hoc test.

To distinguish between astrocytes and microglial cells, analyzed cells were divided into three groups according to their specific staining profile: (i) astrocytes (ACSA-2-positive); (ii) microglial cells (CX3CR1-positve); and (iii) glial cells in association with bacteria (ACSA-2 or CX3CR1-positive plus CFSE-positive). In good agreement with the results shown in Figure 2, in glialcell mono-cultures only the non-encapsulated strain interacted with glial cells (Figure 3A-D). In 10cps $\triangle E F$-infected microglial cell mono-cultures (with and without pre-stimulation with supernatants of uninfected astrocyte mono-cultures) the CFSE-positive cell fraction was associated with a decreased number of microglial cells (Figure 3B,D). Thus, the non-encapsulated mutant most likely interacted with microglial cells. As described above, in case of a $10 \operatorname{cps} \triangle E F$ infection, pretreatment of microglial cell mono-cultures with supernatants of uninfected astrocyte mono-cultures further increased association of glial cells and bacteria (Figure 3B; 3.9\% and 3D; 13.1\%), whereas pretreatment of astrocyte mono-cultures with supernatants of uninfected microglial cell mono-cultures did not influence bacteria-cell association rates of $10 c p s \triangle E F$ (Figure $3 \mathrm{~A} ; 3.3 \%$ and $3 \mathrm{C} ; 2.7 \%$ ).

Astrocytes are known to produce factors interacting with microglial cells. Min et al. [20] detected heat labile compounds in astrocyte supernatants, smaller than $3 \mathrm{kDa}$, influencing microglial cells in case of preventing excessive brain inflammation, whereas in an astrocyte-microglial cell co-cultures studying borna virus infection heat resistant factors activated microglial cells [21]. Moreover, astrocytes secret a variety of proteins like trophic factors, cytokines, chemokines, proteases, and protease inhibitors [31].

One could asked, that supernatants of uninfected glial cells may not contain certain mediators/ components to subsequently efficiently activate the other glial cell type, thus pre-stimulation of glial cell mono-cultures was also performed with supernatants of astrocytes or microglial cells $24 \mathrm{~h}$ pre-infected with $S$. suis $10 \operatorname{cps} \triangle E F$. Surprisingly, no differences in bacteria-cell association were observed between uninfected and pre-infected glial cell supernatants (data not shown). Thus, bacterial pre-infection of astrocytes and microglial cells seems to play a minor role in astrocytes-microglialcell interaction. 


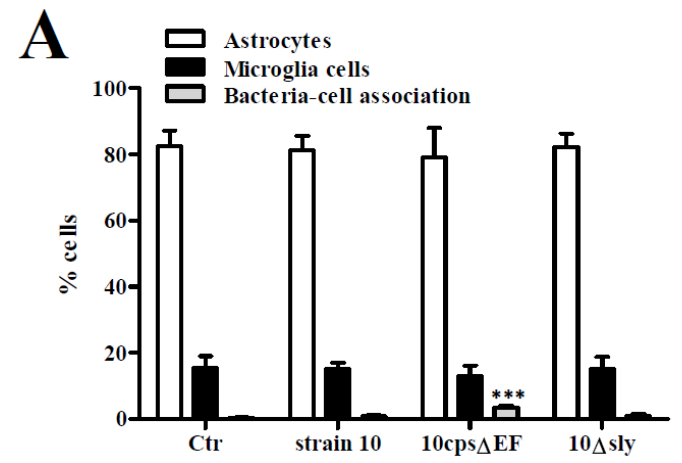

C

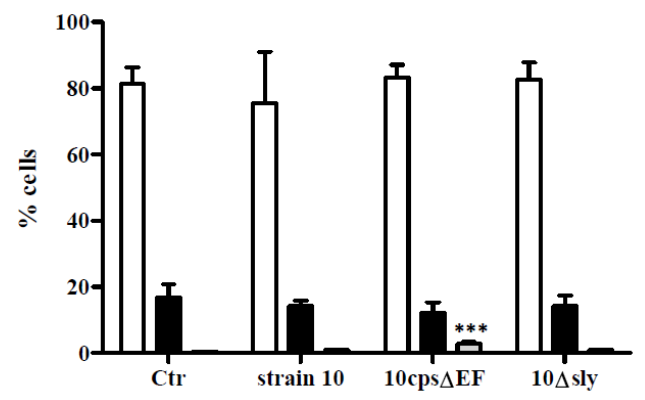

E

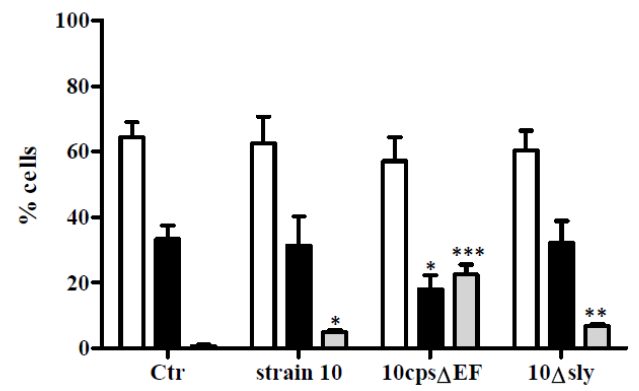

B

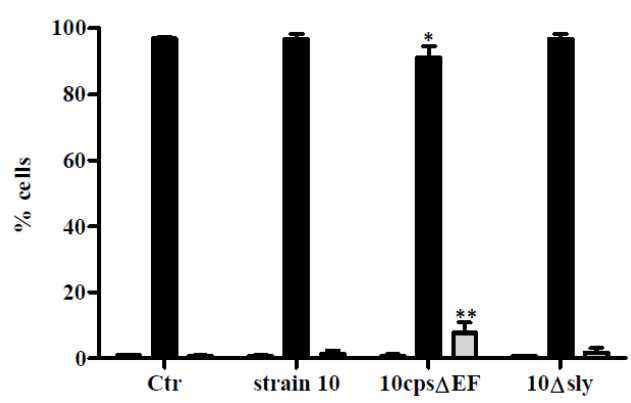

D

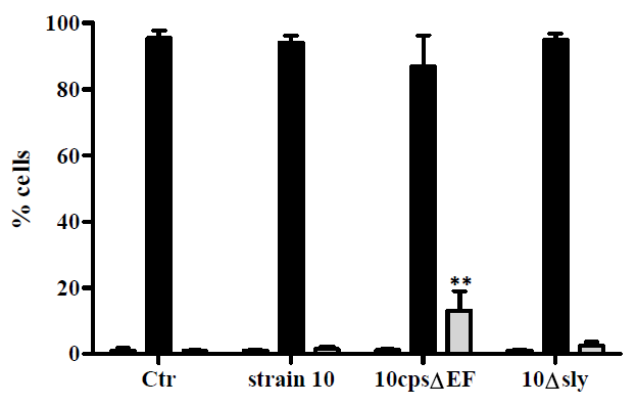

F

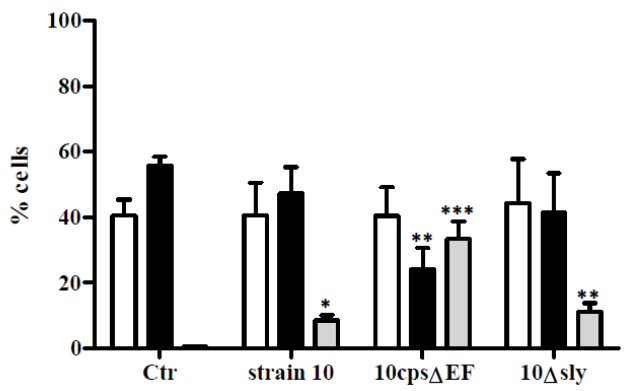

Figure 3. Association of $S$. suis with primary mouse astrocytes and microglial cells. Various glial cell culture systems: (A) astrocyte mono-culture; (B) microglial cell mono-culture; (C) astrocyte mono-culture pre-stimulated with SN of uninfected microglial cell cultures; (D) microglial cell mono-culture pre-stimulated with SN of uninfected astrocyte cultures; (E) astrocyte-microglial cell co-culture (low amount of microglial cells); and (F) astrocyte-microglial cell co-culture (high amount of microglial cells), were infected with CFSE-labeled S. suis strain 10,10cps $\Delta E F$, or $10 \Delta$ sly at a MOI 10:1 for $2 \mathrm{~h}$. Astrocytes and microglial cells were stained for the cell-surface antigens ACSA-2 and CX3CR1, respectively. Relative amounts (\%) of astrocytes (white bars), microglial cells (black bars) and microglial cells/astrocytes in association with bacteria (CFSE-positive, grey bars) were determined by flow cytometry analysis. Results are expressed as means with SD of three independent experiments, and statistically significant differences when compared to uninfected control cells are indicated by * $(p$-value $<0.05),{ }^{* *}(p$-value $<0.01)$, and ${ }^{* * *}(p$-value $<0.001)$, one-way-ANOVA followed by a Dunnett post-hoc test.

In contrast to $10 \operatorname{cps} \triangle E F$, after infection with strain 10 or $10 \Delta$ sly almost no CFSE-positive cell fraction was found in any glial cell mono-culture (with and without pre-incubation with supernatants of uninfected glial cell mono-cultures) (Figure 3A-D). Bacterial replication during infection was 
monitored and confirmed that the observed effects were not due to differences in growth properties of the different strains (Figure S1).

As expected, the amount of glial cells recovered from the uninfected astrocyte-microglial cell co-cultures reflected the initial seeding proportions (Figure 3E; microglial cells: astrocytes 1:2 and 3F; 1:1). In these co-cultures most CFSE-positive cells were observed after infection with 10cps $\triangle E F$, and the fraction of glial cells in association with bacteria increased with the amount of microglial cells added to the co-culture (Figure 3E; 22.6\% and 3F; 33.3\%). Of importance, the amount of astrocytes remained constant, but the percentage of microglial cells significantly decreased in parallel with an increasing CFSE-positive cell fraction after infection with 10cps $\triangle E F$. Thus, microglial cells were the predominant interaction partner for S. suis. Contrary to mono-cultures, in co-cultures both encapsulated S. suis strains were found in association with glial cells, but to a lower extent. The role of suilysin in bacteria-cell interaction is still controversially discussed and highly dependent on the cell type [13,32-36]. Similar to a previous study in a murine microglial cell line [27], but unlike with human astrocytes [30], suilysin seemed to play a minor role in bacteria-cell association, since differences between strain 10 (Figure $3 \mathrm{E} ; 5.0 \%$ and 3F; 8.6\%) and the suilysin-deficient mutant were marginal (Figure 3E; $6.8 \%$ and 3F; $11.1 \%$ ). Since microglial cells are professional phagocytes of the brain, most likely S. suis is phagocytosed by these cells rather than only be attached to them. Hence, a higher association rate of $10 c p s \Delta E F$ with microglial cells in comparison to the tested encapsulated strains is plausible, since the protecting properties of the capsule against phagocytosis are missing. Furthermore, it has been described that primary murine astrocytes are unable to phagocytose even a non-encapsulated strain of S. suis [28]. Taken together, S. suis interacts mainly with microglial cells and S. suis-microglial cell association was facilitated by the presence of astrocytes. Furthermore, association was negatively affected by the capsule, but independent of the production of suilysin.

\subsection{NO Release by Primary Mouse Glial Cells after Infection with S. suis}

Reactive glial cells can produce a variety of second mediators, such as cytokines, neurotransmitters or nitic oxide (NO) that are involved in neuroinflammatory and -degenerative processes [37]. To analyze NO release by astrocytes and microglial cells as well as to study the possible interaction of both glial cell types, the glial cell cultures introduced above were infected at a MOI of 10:1 for 2,7 and $24 \mathrm{~h}$. At early time points post infection ( 2 and $7 \mathrm{~h}$ post infection (hpi)) no significant NO levels were detectable in any glial cell culture (Figure 4). As shown in Figure 4A,B even after 24 hpi no significant NO release was observed by mono-cultured astrocytes or microglial cells infected with S. suis. Interestingly, also pre-stimulation of astrocytes with supernatants of uninfected microglial cell mono-cultures failed to induce NO release (Figure 4C), but an efficient NO release was measured $24 \mathrm{hpi}$ in microglial cell cultures pre-incubated with supernatants of uninfected astrocyte mono-cultures (Figure 4D). The capsular mutant 10cps $\triangle E F$ induced the highest NO levels when compared to uninfected microglial cells pre-incubated with the supernatant of uninfected astrocytes (control, Ctr) (3.2-fold). Both encapsulated S. suis strains 10 and 10 $\Delta$ sly showed a 2.7-fold higher NO release. To test the direct effect of astrocytes on the NO release by microglial cells, both co-culture systems were used. 24 hpi significantly higher NO levels were measured in co-cultures infected with $S$. suis strain $10 \operatorname{cps} \triangle E F$ (Figure $4 \mathrm{E}, \mathrm{F}$ ). In accordance to the results described above, the level of released $\mathrm{NO}$ in the co-cultures was dependent on the number of microglial cells added to the system. Strain $10 \mathrm{cps} \triangle E F$ induced a 3.0-fold (low amount of microglial cells) and 3.3-fold (high amount of microglial cells) NO release, respectively, when compared to uninfected co-cultures. Thus, NO seems to be mainly produced by microglial cells, but astrocytes are indirectly (soluble factors in the supernatant) or directly (cellular contact) crucial for a sufficient NO release by microglial cells after infection with S. suis. In fact, microglial cells are accepted as the main glial cell type responsible for NO release, e.g., upon lipopolysaccharide (LPS)stimulation or S. suis infection [37]. Nevertheless, astrocytes appear to be capable of expressing inducible nitric oxide synthase (iNOS) $[28,37]$. In addition, in a previous study, NO release by LPS-stimulated microglial cells was described to be enhanced in the presence of astrocytes [37]. 
A

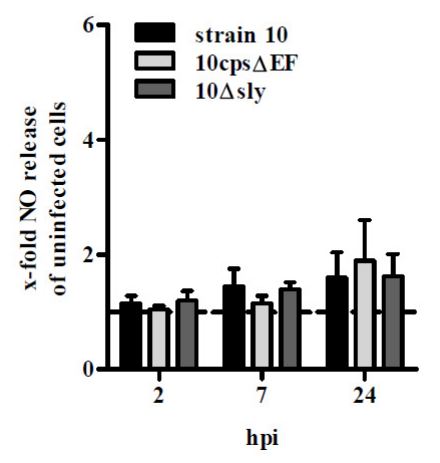

C

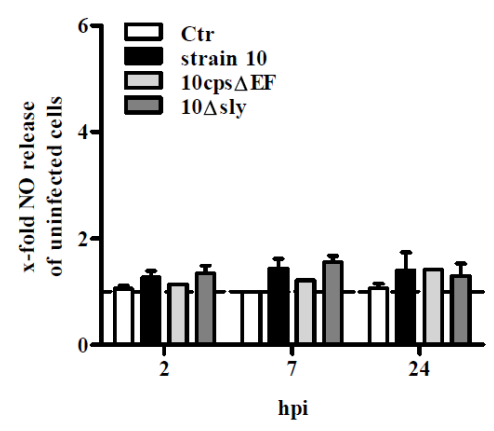

$\mathbf{E}$

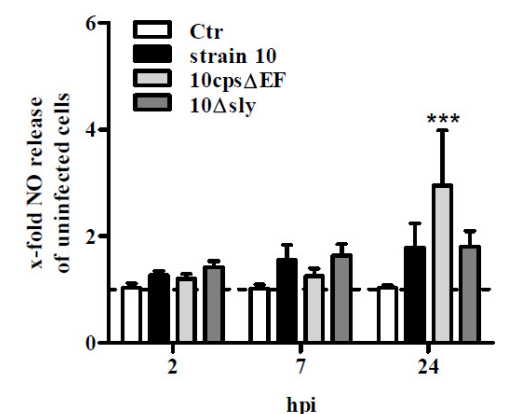

B

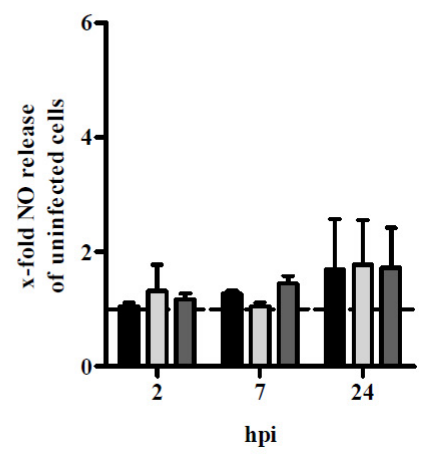

D

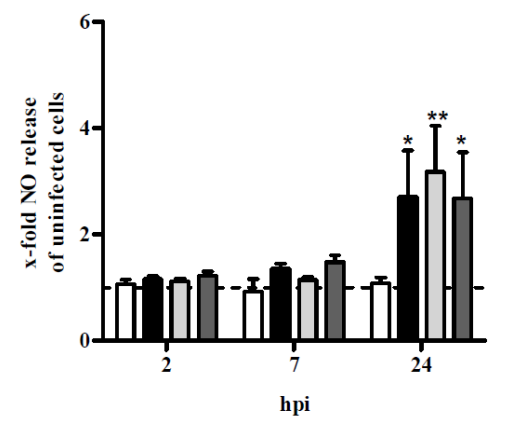

F

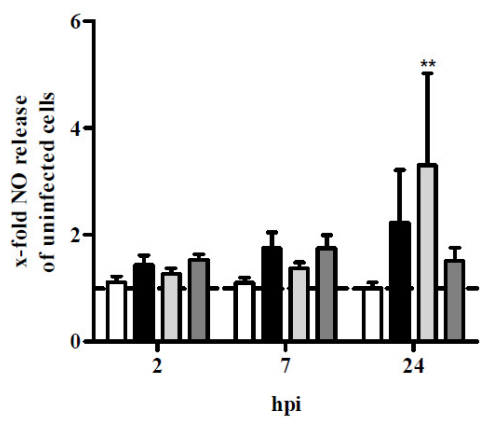

Figure 4. NO release by primary mouse glial cell cultures after infection with S. suis. Various glial cell culture systems: (A) astrocyte mono-culture; (B) microglial cell mono-culture; (C) astrocyte mono-culture pre-stimulated with $\mathrm{SN}$ of uninfected microglial cell cultures; (D) microglial cell mono-culture pre-stimulated with SN of uninfected astrocyte cultures; (E) astrocyte-microglial cell co-culture (low amount of microglial cells); and (F) astrocyte-microglial cell co-culture (high amount of microglial cells), were infected with S. suis strain 10,10cps $\Delta E F$, or 10 $\Delta$ sly at a MOI of 10:1 for 2, 7, and $24 \mathrm{~h}$ (hpi). Nitirc oxide (NO) release was measured using Griess reagent. All results are expressed as $x$-fold NO release normalized to uninfected astrocytes or microglial cells which were not pre-incubated with SN (dashed line). In the co-culture system values were normalized to uninfected astrocytes. Uninfected astrocytes or microglial cells pre-incubated with $\mathrm{SN}$ only or co-cultured served as further controls (Ctr, C-F). Results are expressed as means with SD of four (A + B) or six (C-F) independent experiments and statistically significant differences when compared to uninfected astrocytes and microglial cells $(\mathbf{A}, \mathbf{B})$, uninfected astrocytes and microglial cells pre-incubated with supernatants $(\mathbf{C}, \mathbf{D})$ or astrocyte-microglial cell co-cultures $(\mathbf{E}, \mathbf{F})$ are indicated by * $(p$-value $<0.05),{ }^{* *}(p$-value $<0.01)$, and $* * *$ ( $p$-value $<0.001)$, one-way-ANOVA followed by Dunnett post-hoc test. 
In contrast to $10 \operatorname{cps} \Delta E F$, the increase of the $N O$ release caused by the encapsulated S. suis strains in the co-cultures, was small and failed to reach statistical significance (Figure 4E,F). Differences observed between encapsulated and non-encapsulated $S$. suis strains in the ability to induce NO were not related to differences in growth, since replication rates of all tested strains were similar at all tested time points (Figure S1). Nevertheless, as described above encapsulated S. suis strains were able to induce a NO release by microglial cells when pre-incubated with supernatants of uninfected astrocyte mono-cultures (Figure 4D). This might be due to the fact that supernatants of astrocyte mono-cultures for pre-incubation were harvested after 3-5 days after cultivation whereas co-cultures were only incubated for 1 day. Thus, soluble factors stimulating NO release by microglial cells might be higher concentrated in supernatants of astrocyte mono-cultures than in the co-cultures.

The masking effect of the capsule, which might hide cell surface structures crucial for microglial cell activation, may explain why the non-encapsulated mutant of $S$. suis induced the highest NO levels [27,28]. For instance, cellwall components such as lipoteichoic acid (LTA) of Gram-positive bacteria are known to activate NO in microglial cells [38]. Nevertheless, knowledge of receptors and mechanisms involved in the activation of microglial cells infected with $S$. suis is limited. First results indicate that Toll-like receptor 2 (TLR-2), nucleotide oligomerization domain 2 (NOD)-2 and signal transducer and activator of transcription 3 (STAT3) signaling are involved in interaction with $S$. suis as well as mitogen-activated protein kinases (MAPK) signaling events and nuclear factor kappa-light-chain-enhancer of activated B cells (NF-KB) activation $[27,29]$. Since we found a higher association rate as well as higher levels of NO in microglial cells after infection with the non-encapsulated S. suis strain, one could speculate that phagocytosis of S. suis might be necessary to subsequent induce sufficient NO release by microglial cells. In our glial cell cultures astrocytes showed an important supportive function to stimulate phagocytosis and NO release by microglial cells. Thus, both glial cell types seem to interact with each other during infection with S. suis.

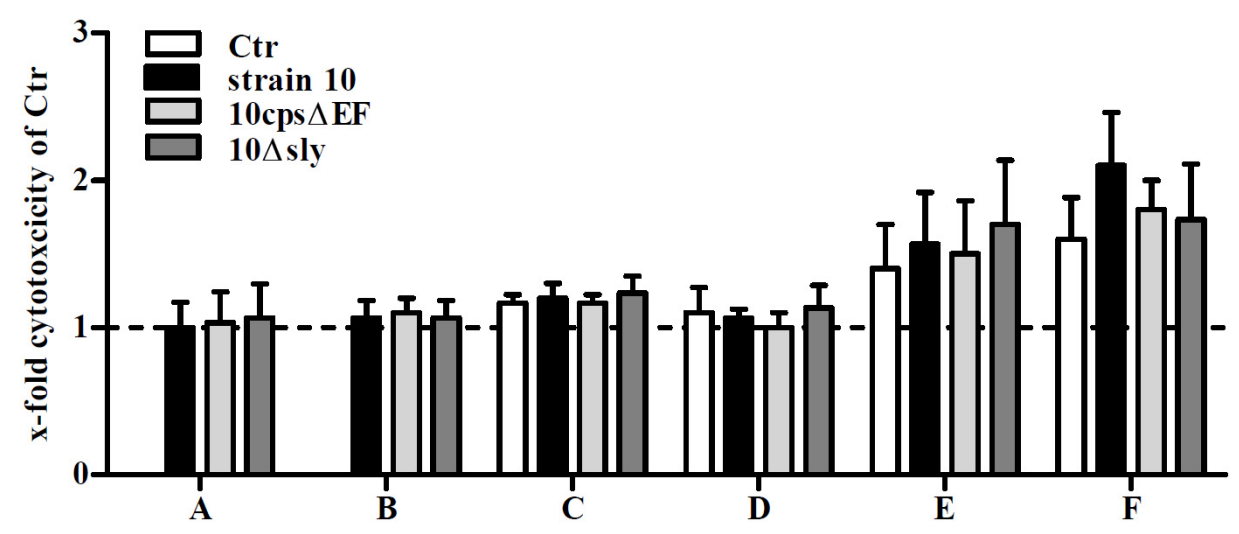

Figure 5. Cell viability of primary mouse glial cell cultures after infection with $S$. suis. Various glial cell culture systems: (A) astrocyte mono-culture; (B) microglial cell mono-culture; (C) astrocyte mono-culture pre-stimulated with SN of uninfected microglial cell cultures; (D) microglial cell mono-culture pre-stimulated with SN of uninfected astrocyte cultures; (E) astrocyte-microglial cell co-culture (low amount of microglial cells); and (F) astrocyte-microglial cell co-culture (high amount of microglial cells), were infected with $S$. suis strain 10,10cps $\Delta E F$, or 10 $\Delta$ sly at a MOI 10:1. 24 h post infection cell viability was determined by standard lactate dehydrogenase (LDH) release assay. Results are expressed as $\mathrm{x}$-fold cytotoxicity normalized to uninfected astrocytes and microglial cells which were not pre-incubated with SN (dashed line). In the co-culture system values were normalized to uninfected astrocytes. Uninfected astrocytes or microglial cells pre-stimulated with SN only or co-cultured served as further controls (Ctr, C-F). Results are expressed as means with SD of three independent experiments. No significant differences when compared to uninfected astrocytes and microglial cells (A,B), uninfected astrocytes and microglial cells pre-incubated with supernatants (C,D) or astrocyte-microglial cell co-cultures $(\mathbf{E}, \mathbf{F})$ were found, one-way-ANOVA followed by a Dunnett post-hoc test. 
NO can have either beneficial (cytoprotective) or detrimental (cytotoxic) effects [39]. To test cytotoxic effects by the infection, which may depend on the NO release, a classical LDH release test was performed $24 \mathrm{hpi}$ (Figure 5). Only moderate cytotoxic effects were observed in both co-culture systems without infection reflecting viable astrocytes and microglial cells and after infection with S. suis. In good agreement, cytotoxic effects lower than $1.5 \%$ were found in primary astrocyte cultures $24 \mathrm{~h}$ after infection with $S$. suis [28]. In contrast, S. suis infection led to a time-dependent increase in cytotoxicity levels in BV2 microglial cell line [29]. Interestingly, cytotoxicity was independent of the presence of suilysin, because the suilysin-positive strain 10 and its suilysin-deficient mutant behaved the same way. Nevertheless, since no LDH release was found in S. suis-infected microglial cell mono-cultures pre-stimulated with supernatants of uninfected astrocytes, which showed a significant NO release after infection, no correlation between cytotoxic effects and NO could be found.

\section{Experimental Section}

\subsection{Bacterial Strains and Growth Conditions}

S. suis strain 10 is a virulent serotype 2 strain (sequence type 1 ) that has previously been used for experimental infections of piglets and for generation of isogenic mutants [6,40-42]. It expresses the virulence-associated muramidase-released protein (MRP), the extracellular factor (EF) and suilysin [43]. The capsule-deficient isogenic mutant $10 \operatorname{cps} \triangle E F$ is attenuated in virulence. It was originally generated through insertion of a spectinomycin-resistance cassette in the genes cps2E and cps2F [6]. The suilysin-deficient mutant, strain $10 \Delta$ sly, was constructed by insertion of an erythromycin cassette in the sly gene of strain 10 using the plasmid pBlue/sly/erm as previously described [33]. Streptococci were grown on Columbia blood agar plates for $16 \mathrm{~h}$ or in Bacto ${ }^{\mathrm{TM}}$ Todd Hewitt broth (THB) to logarithmic growth phase (optical density at $600 \mathrm{~nm}$ of 0.6 ) at $37^{\circ} \mathrm{C}$ under aerobic conditions.

\subsection{Isolation and Cultivation of Mixed Glial Cell Cultures}

Mice were cared for in accordance with the principles outlined in the European Convention for the Protection of Vertebrate Animals Used for Experimental and Other Scientific Purposes (European Treaty Series, no. 123 [44]). Mixed glial cells were isolated from brains of newborn (0-2 days postnatal) wild-type C57BL/ 6 mice and cultivated as previously described [45]. Briefly, meninges of murine brains were removed, whole brains were treated with $0.25 \%$ trypsine (Biochrom, Berlin, Germany; $2.5 \%$ solution) in HBSS (Sigma-Aldrich, Taufkirchen, Germany) for $10 \mathrm{~min}$ at $37^{\circ} \mathrm{C}$, DNAse (Sigma-Aldrich, Taufkirchen, Germany) was added in a concentration of $0.4 \mathrm{mg} / \mathrm{mL}$, and brains were minced by pipetting up and down with a $10 \mathrm{~mL}$ pipette. After centrifugation $(750 \times g, 8 \mathrm{~min})$ and removal of supernatant cells of two brains were seeded into a $75 \mathrm{~cm}^{2}$ cell culture flask and cultured in DMEM $4.5 \mathrm{~g} / \mathrm{L}$ glucose (Gibco, Karlsruhe, Germany) supplemented with $10 \%$ heat-inactivated FCS, $5 \mathrm{mM}$ glutamine, and $100 \mathrm{U} / \mathrm{mL}$ penicillin and $100 \mu \mathrm{g} / \mathrm{mL}$ streptomycin at $37^{\circ} \mathrm{C}+5 \% \mathrm{CO}_{2}$ for 10 days or until confluency of the cell layer was reached.

\subsection{Cultivation and Separation of Astrocytes and Microglial Cells}

After 10 days of mixed glial cell cultivation the medium was changed to antibiotic-free conditions. Supernatants of fibroblast cell line L929 were added to a final volume of $30 \%$ [v/v]. After 5 days of stimulation microglial cells were harvested by shaking ( $150 \mathrm{rpm} / \mathrm{min}$ ) for $30 \mathrm{~min}$ at $37^{\circ} \mathrm{C}$ and seeded into 24 - or 6-well plates in a concentration of $1 \times 10^{5}$ cells/well or $4 \times 10^{5}$ cells/well for microglial cell mono-cultures, respectively. The remaining glial cell culture was again stimulated with supernatants of fibroblast cell line L929 and harvesting of microglial cells was repeated at most five times. For astrocyte cultures the dividing cells in the mixed glial cell cultures were removed by treatment with $100 \mu \mathrm{m}$ AraC for $72 \mathrm{~h}$ (Sigma, Taufkirchen, Germany) as described elsewhere [46]. Subsequently, astrocytes were harvested by trypsin digestion and seeded into 24- or 6-well plates in a concentration of $1 \times 10^{5}$ cells/well or $4 \times 10^{5}$ cells/well for astrocyte mono-cultures, respectively. 
For co-cultivation of astrocytes and microglial cells, $1 \times 10^{5}$ or $4 \times 10^{5}$ astrocytes/well were seeded into 24- or 6-well plates, respectively. After $24 \mathrm{~h}$ of incubation at $37^{\circ} \mathrm{C}+5 \% \mathrm{CO}_{2} 1$ or $2 \times 10^{5}$ microglial cells/well (24-well plate; low and high microglial cell number, respectively) and 4 or $8 \times 10^{5}$ microglial cells/well (6-well plate, low or high microglial cell number, respectively) were seeded on top of the astrocytes and incubated for further $24 \mathrm{~h}$ at $37^{\circ} \mathrm{C}+5 \% \mathrm{CO}_{2}$.

\subsection{Pre-incubation of Astrocytes and Microglial Cells with Cell Supernatants}

Astrocytes and microglial cells were pre-incubated with supernatants of microglial cell and astrocyte mono-cultures, respectively, to investigate the influence of soluble factors on activity of these two cell types. Supernatants of both cell types were harvested after 3-5 days of cultivation. Cells were pre-incubated with supernatants for $24 \mathrm{~h}$ at $37^{\circ} \mathrm{C}+5 \% \mathrm{CO}_{2}$.

\subsection{Flow Cytometry Characterization of Astrocytes and Microglial Cells}

Astrocytes and astrocyte-microglial cell co-cultures were harvested by trypsin treatment and microglial cells by scraping. Approximately $3 \times 10^{5}$ cells of each cell type were stained with antibodies against extracellular markers: anti-CD11b-APC, anti-CD11c-PE, anti-CD45-FITC, anti-ACSA-2-PE (Miltenyi Biotec, Bergisch Gladbach, Germany) and anti-CX3CR1-FITC (BioLegend, Koblenz, Germany) as recommended by the manufacturer. For intracellular staining of CD68 and GFAP cells were treated with the Intracellular Fixation \& Permeabilization Buffer Set (Affymetrix eBioscience, Frankfurt am Main, Germany) as recommended by the manufacturer and subsequently stained with anti-CD68-APC and anti-GFAP-PE (Miltenyi Biotec, Bergisch Gladbach, Germany) as recommended by the manufacturer. Cells were measured with a Guava ${ }^{\circledR}$ easyCyte flow cytometer (Merck Millipore, Darmstadt, Germany) and analyzed with the guava InCyte ${ }^{\mathrm{TM}}$ Software. For each sample 5000 events were acquired and analyzed in a dot plot or histogram.

\subsection{Flow Cytometry Analysis of Bacteria-Cell-Association}

For this purpose, streptococcal strains were labeled with the carboxyfluorescein succinimidyl ester. (CFSE) using CellTrace ${ }^{\mathrm{TM}}$ CFSE Cell Proliferation Kit (Invitrogen, Karlsruhe, Germany). Labeling was performed as described [32].

Mono- or co-cultured cells with or without pre-stimulation with cell supernatants were infected with CFSE-labeled S. suis with a multiplicity of infection (MOI) of 10:1 (bacterium:cell) for $2 \mathrm{~h}$. Subsequently, the supernatant was removed and the cells were washed three times with PBS. Astrocytes and astrocyte-microglial cell co-cultures were harvested by trypsin treatment and microglial cells by scraping. Following, astrocytes were stained with anti-ASCA-2-PE (Miltenyi Biotec, Bergisch Gladbach, Germany) and microglial cells with anti-CX3CR1-APC (BioLegend, Koblenz, Germany) as described above. Cells were measured with a Guava ${ }^{\circledR}$ easyCyte flow cytometer (Merck Millipore, Darmstadt, Germany) and analyzed with the guava InCyte ${ }^{\mathrm{TM}}$ Software. Furthermore, glial cells in association with bacteria (ACSA-2 or CX3CR1-positive plus CFSE-positive) were determined. For each sample 5000 events were acquired and analyzed in a dot plot or histogram.

\subsection{NO Measurement}

Mono- or co-cultured cells with or without pre-incubation with cell supernatants were infected with S. suis with a MOI of 10:1 (bacterium:cell) for 2, 7 or $24 \mathrm{~h}$. Nitrite was measured in cell supernatants using Griess reaction (Thermo Fisher Scientific, Darmstadt, Germany) as recommended by the manufacturer.

\subsection{Evaluation of Cytotoxic Effects}

Cytotoxicity was detected by lactate dehydrogenase (LDH) release assay after $24 \mathrm{~h}$ of infection as described previously [47]. 


\subsection{Statistical Analysis}

If not stated otherwise experiments were performed at least three times and one-way analysis of variance (ANOVA) followed by a Dunnett or Turkey post-hoc test was used. Probabilities lower than 0.05 were considered significant $\left(p<0.05^{*}, p<0.01^{* *}\right.$ and $\left.p<0.001^{* * *}\right)$.

\section{Conclusions}

In conclusion, S. suis interacts poorly with mono-cultured microglial cells and astrocytes, but infection of microglial cells co-cultured with (supernatants of) astrocytes leads to the induction of NO release and S. suis is found in association with these cells. Bacteria-cell association as well as NO release by microglial cells is efficiently enhanced in the presence of astrocytes and influenced by the presence of the capsule, since the non-encapsulated mutant shows the highest effects. These results underline the important function of astrocytes during bacterial infection of the CNS and the need to investigate complex in vitro models. Further experiments are needed to identify bacterial and host cell factors involved in the crosstalk between microglial cells and astrocytes to analyze the underlying mechanisms of astrocyte-microglialcell interaction in S. suis CNS infections.

Supplementary Materials: The following are available online at www.mdpi.com/2076-0817/5/2/43/s1, Figure S1: Replication rates of $S$. suis strain 10,10 $\Delta$ sly and $10 \operatorname{cps} \Delta E F$ on primary mouse glial cell cultures.

Acknowledgments: We thank H. Smith (DLO-Lelystad, Netherlands) for S. suis strains 10 and $10 \mathrm{cps} \Delta E F$. Experiments were supported by Saskia Kreis (Institute for Microbiology, University of Veterinary Medicine Hannover). This study was supported by the Niedersachsen Research Network on Neuroinfectiology of the Ministry of Science and Culture of Lower Saxony (N-RENNT) and by the German Federal Ministry for Research and Education (BMBF) within the Helmholtz-CAS-Joint Research Group ZooStrep (HCJRG-116).

Author Contributions: Jana Seele contributed to study design, conducted the experiments and drafted the manuscript. Peter Valentin-Weigand contributed in study design and manuscript revision. Roland Nau, Martin Stangel and Chittappen K. Prajeeth supported establishment of mixed glial cell preparations and revised the manuscript. Maren Seitz conceived the study, designed and conducted experiments and drafted the manuscript. All authors read and approved the final manuscript.

Conflicts of Interest: The authors declare that they have no conflicts of interests.

\section{References}

1. Reams, R.Y.; Glickman, L.T.; Harrington, D.D.; Thacker, H.L.; Bowersock, T.L. Streptococcus suis infection in swine: A retrospective study of 256 cases. Part ii. Clinical signs, gross and microscopic lesions, and coexisting microorganisms. J. Vet. Diagn. Investig. 1994, 6, 326-334. [CrossRef] [PubMed]

2. Straw, B.E. Diseases of Swine, 9th ed.; Blackwell Publisher: Ames, IA, USA, 2006.

3. Huong, V.T.; Ha, N.; Huy, N.T.; Horby, P.; Nghia, H.D.; Thiem, V.D.; Zhu, X.; Hoa, N.T.; Hien, T.T.; Zamora, J.; et al. Epidemiology, clinical manifestations, and outcomes of Streptococcus suis infection in humans. Emerg. Infect. Dis. 2014, 20, 1105-1114. [CrossRef] [PubMed]

4. Baums, C.G.; Valentin-Weigand, P. Surface-associated and secreted factors of Streptococcus suis in epidemiology, pathogenesis and vaccine development. Anim. Health Res. Rev. 2009, 10, 65-83. [CrossRef] [PubMed]

5. Charland, N.; Harel, J.; Kobisch, M.; Lacasse, S.; Gottschalk, M. Streptococcus suis serotype 2 mutants deficient in capsular expression. Microbiology 1998, 144, 325-332. [CrossRef] [PubMed]

6. Smith, H.E.; Damman, M.; van der Velde, J.; Wagenaar, F.; Wisselink, H.J.; Stockhofe-Zurwieden, N.; Smits, M.A. Identification and characterization of the cps locus of Streptococcus suis serotype 2: The capsule protects against phagocytosis and is an important virulence factor. Infect. Immun. 1999, 67, 1750-1756. [PubMed]

7. Chabot-Roy, G.; Willson, P.; Segura, M.; Lacouture, S.; Gottschalk, M. Phagocytosis and killing of Streptococcus suis by porcine neutrophils. Microb. Pathog. 2006, 41, 21-32. [CrossRef] [PubMed]

8. Seitz, M.; Beineke, A.; Singpiel, A.; Willenborg, J.; Dutow, P.; Goethe, R.; Valentin-Weigand, P.; Klos, A.; Baums, C.G. Role of capsule and suilysin in mucosal infection of complement-deficient mice with Streptococcus suis. Infect. Immun. 2014, 82, 2460-2471. [CrossRef] [PubMed] 
9. Fittipaldi, N.; Segura, M.; Grenier, D.; Gottschalk, M. Virulence factors involved in the pathogenesis of the infection caused by the swine pathogen and zoonotic agent Streptococcus suis. Future Microbiol. 2012, 7 , 259-279. [CrossRef] [PubMed]

10. Gottschalk, M.G.; Lacouture, S.; Dubreuil, J.D. Characterization of Streptococcus suis capsular type 2 haemolysin. Microbiology 1995, 141, 189-195. [CrossRef] [PubMed]

11. Benga, L.; Friedl, P.; Valentin-Weigand, P. Adherence of Streptococcus suis to porcine endothelial cells. J. Vet. Med. B Infect. Dis. Vet. Public Health 2005, 52, 392-395. [CrossRef] [PubMed]

12. Charland, N.; Nizet, V.; Rubens, C.E.; Kim, K.S.; Lacouture, S.; Gottschalk, M. Streptococcus suis serotype 2 interactions with human brain microvascular endothelial cells. Infect. Immun. 2000, 68, 637-643. [CrossRef] [PubMed]

13. Vanier, G.; Segura, M.; Friedl, P.; Lacouture, S.; Gottschalk, M. Invasion of porcine brain microvascular endothelial cells by Streptococcus suis serotype 2. Infect. Immun. 2004, 72, 1441-1449. [CrossRef] [PubMed]

14. Vanier, G.; Segura, M.; Gottschalk, M. Characterization of the invasion of porcine endothelial cells by Streptococcus suis serotype 2. Can. J. Vet. Res. 2007, 71, 81-89. [PubMed]

15. Tenenbaum, T.; Papandreou, T.; Gellrich, D.; Friedrichs, U.; Seibt, A.; Adam, R.; Wewer, C.; Galla, H.J.; Schwerk, C.; Schroten, H. Polar bacterial invasion and translocation of Streptococcus suis across the blood-cerebrospinal fluid barrier in vitro. Cell. Microbiol. 2009, 11, 323-336. [CrossRef] [PubMed]

16. Sofroniew, M.V. Astrocyte barriers to neurotoxic inflammation. Nat. Rev. Neurosci. 2015, 16, $249-263$. [CrossRef] [PubMed]

17. Kimelberg, H.K.; Nedergaard, M. Functions of astrocytes and their potential as therapeutic targets. Neurotherapeutics 2010, 7, 338-353. [PubMed]

18. Stoner, T.D.; Weston, T.A.; Trejo, J.; Doran, K.S. Group B streptococcal infection and activation of human astrocytes. PLoS ONE 2015, 10, e0128431. [CrossRef] [PubMed]

19. Olson, J.K.; Miller, S.D. Microglia initiate central nervous system innate and adaptive immune responses through multiple tlrs. J. Immunol. 2004, 173, 3916-3924. [CrossRef] [PubMed]

20. Min, K.J.; Yang, M.S.; Kim, S.U.; Jou, I.; Joe, E.H. Astrocytes induce hemeoxygenase-1 expression in microglia: A feasible mechanism for preventing excessive brain inflammation. J. Neurosci. 2006, 26, 1880-1887. [CrossRef] [PubMed]

21. Ovanesov, M.V.; Ayhan, Y.; Wolbert, C.; Moldovan, K.; Sauder, C.; Pletnikov, M.V. Astrocytes play a key role in activation of microglia by persistent borna disease virus infection. J. Neuroinflammation 2008, 5, 50. [CrossRef] [PubMed]

22. Carson, M.J.; Thrash, J.C.; Walter, B. The cellular response in neuroinflammation: The role of leukocytes, microglia and astrocytes in neuronal death and survival. Clin. Neurosci. Res. 2006, 6, 237-245. [CrossRef] [PubMed]

23. Cooley, I.D.; Chauhan, V.S.; Donneyz, M.A.; Marriott, I. Astrocytes produce il-19 in response to bacterial challenge and are sensitive to the immunosuppressive effects of this il-10 family member. Glia 2014, 62, 818-828. [CrossRef] [PubMed]

24. Liu, X.; Chauhan, V.S.; Young, A.B.; Marriott, I. Nod2 mediates inflammatory responses of primary murine glia to Streptococcus pneumoniae. Glia 2010, 58, 839-847. [PubMed]

25. Hansson, E.; Ronnback, L. Glial neuronal signaling in the central nervous system. FASEB J. 2003, 17, 341-348. [CrossRef] [PubMed]

26. Bezzi, P.; Domercq, M.; Brambilla, L.; Galli, R.; Schols, D.; de Clercq, E.; Vescovi, A.; Bagetta, G.; Kollias, G.; Meldolesi, J.; et al. CXCR4-activated astrocyte glutamate release via tnfalpha: Amplification by microglia triggers neurotoxicity. Nat. Neurosci. 2001, 4, 702-710. [CrossRef] [PubMed]

27. Dominguez-Punaro Mde, L.; Segura, M.; Contreras, I.; Lachance, C.; Houde, M.; Lecours, M.P.; Olivier, M.; Gottschalk, M. In vitro characterization of the microglial inflammatory response to Streptococcus suis, an important emerging zoonotic agent of meningitis. Infect Immun. 2010, 78, 5074-5085. [CrossRef] [PubMed]

28. Zheng, H.; Punaro, M.C.; Segura, M.; Lachance, C.; Rivest, S.; Xu, J.; Houde, M.; Gottschalk, M. Toll-like receptor 2 is partially involved in the activation of murine astrocytes by Streptococcus suis, an important zoonotic agent of meningitis. J. Neuroimmunol. 2011, 234, 71-83. [CrossRef] [PubMed]

29. Zheng, H.; Sun, H.; Dominguez-Punaro Mde, L.; Bai, X.; Ji, S.; Segura, M.; Xu, J. Evaluation of the pathogenesis of meningitis caused by Streptococcus suis sequence type 7 using the infection of bv2 microglial cells. J. Med. Microbiol. 2013, 62, 360-368. [CrossRef] [PubMed] 
30. Auger, J.P.; Christodoulides, M.; Segura, M.; Xu, J.; Gottschalk, M. Interactions of Streptococcus suis serotype 2 with human meningeal cells and astrocytes. BMC Res. Notes 2015, 8, 607. [CrossRef] [PubMed]

31. Dowell, J.A.; Johnson, J.A.; Li, L. Identification of astrocyte secreted proteins with a combination of shotgun proteomics and bioinformatics. J. Proteome Res. 2009, 8, 4135-4143. [CrossRef] [PubMed]

32. Seitz, M.; Baums, C.G.; Neis, C.; Benga, L.; Fulde, M.; Rohde, M.; Goethe, R.; Valentin-Weigand, P. Subcytolytic effects of suilysin on interaction of Streptococcus suis with epithelial cells. Vet. Microbiol. 2013, 167, 584-591. [CrossRef] [PubMed]

33. Benga, L.; Fulde, M.; Neis, C.; Goethe, R.; Valentin-Weigand, P. Polysaccharide capsule and suilysin contribute to extracellular survival of Streptococcus suis co-cultivated with primary porcine phagocytes. Vet. Microbiol. 2008, 132, 211-219. [CrossRef] [PubMed]

34. Segura, M.; Gottschalk, M. Streptococcus suis interactions with the murine macrophage cell line j774: Adhesion and cytotoxicity. Infect. Immun. 2002, 70, 4312-4322. [CrossRef] [PubMed]

35. Norton, P.M.; Rolph, C.; Ward, P.N.; Bentley, R.W.; Leigh, J.A. Epithelial invasion and cell lysis by virulent strains of Streptococcus suis is enhanced by the presence of suilysin. FEMS Immunol Med. Microbiol. 1999, 26, 25-35. [CrossRef] [PubMed]

36. Tenenbaum, T.; Adam, R.; Eggelnpohler, I.; Matalon, D.; Seibt, A.; Novotny, G.E.K.; Galla, H.J.; Schroten, H. Strain-dependent disruption of blood-cerebrospinal fluid barrier by Streptoccocus suis in vitro. FEMS Immunol. Med. Microbiol. 2005, 44, 25-34. [CrossRef] [PubMed]

37. Sola, C.; Casal, C.; Tusell, J.M.; Serratosa, J. Astrocytes enhance lipopolysaccharide-induced nitric oxide production by microglial cells. Eur. J. Neurosci. 2002, 16, 1275-1283. [CrossRef] [PubMed]

38. Chien, H.F.; Yeh, K.Y.; Jiang-Shieh, Y.F.; Wei, I.H.; Chang, C.Y.; Chang, M.L.; Wu, C.H. Signal transduction pathways of nitric oxide release in primary microglial culture challenged with gram-positive bacterial constituent, lipoteichoic acid. Neuroscience 2005, 133, 423-436. [CrossRef] [PubMed]

39. Wiley, J. The many faces of nitric oxide: Cytotoxic, cytoprotective or both. Neurogastroenterol. Motil. 2007, 19, 541-544. [CrossRef] [PubMed]

40. Baums, C.G.; Kaim, U.; Fulde, M.; Ramachandran, G.; Goethe, R.; Valentin-Weigand, P. Identification of a novel virulence determinant with serum opacification activity in Streptococcus suis. Infect. Immun. 2006, 74, 6154-6162. [CrossRef] [PubMed]

41. Baums, C.G.; Kock, C.; Beineke, A.; Bennecke, K.; Goethe, R.; Schroder, C.; Waldmann, K.H.; Valentin-Weigand, P. Streptococcus suis bacterin and subunit vaccine immunogenicities and protective efficacies against serotypes 2 and 9. Clin. Vaccine Immunol. 2009, 16, 200-208. [CrossRef] [PubMed]

42. De Greeff, A.; Buys, H.; Verhaar, R.; Dijkstra, J.; van Alphen, L.; Smith, H.E. Contribution of fibronectin-binding protein to pathogenesis of Streptococcus suis serotype 2. Infect. Immun. 2002, 70, 1319-1325. [CrossRef] [PubMed]

43. Smith, H.E.; Vecht, U.; Wisselink, H.J.; Stockhofe-Zurwieden, N.; Biermann, Y.; Smits, M.A. Mutants of Streptococcus suis types 1 and 2 impaired in expression of muramidase-released protein and extracellular protein induce disease in newborn germfree pigs. Infect. Immun. 1996, 64, 4409-4412. [PubMed]

44. Council of Europe. Details of Treaty No.123. Available online: http://conventions.coe.int/treaty/en/ treaties/html/123.htm (accessed on 7 June 2016).

45. Redlich, S.; Ribes, S.; Schutze, S.; Eiffert, H.; Nau, R. Toll-like receptor stimulation increases phagocytosis of Cryptococcus neoformans by microglial cells. J. Neuroinflammation 2013, 10, 71. [CrossRef] [PubMed]

46. Sun, H.; Benardais, K.; Stanslowsky, N.; Thau-Habermann, N.; Hensel, N.; Huang, D.; Claus, P.; Dengler, R.; Stangel, M.; Petri, S. Therapeutic potential of mesenchymal stromal cells and msc conditioned medium in amyotrophic lateral sclerosis (als)-in vitro evidence from primary motor neuron cultures, nsc-34 cells, astrocytes and microglia. PLoS ONE 2013, 8, e72926. [CrossRef] [PubMed]

47. Benga, L.; Goethe, R.; Rohde, M.; Valentin-Weigand, P. Non-encapsulated strains reveal novel insights in invasion and survival of Streptococcus suis in epithelial cells. Cell. Microbiol. 2004, 6, 867-881. [CrossRef] [PubMed]

(C) 2016 by the authors; licensee MDPI, Basel, Switzerland. This article is an open access article distributed under the terms and conditions of the Creative Commons Attribution (CC-BY) license (http:/ / creativecommons.org/licenses/by/4.0/). 